\title{
Identification of Targetable ALK Rearrangements in Pancreatic Ductal Adenocarcinoma
}

\author{
Aatur D. Singhi, MD, PhD ${ }^{\mathrm{a},{ }^{*}}$; Siraj M. Ali, MD, PhD ${ }^{\mathrm{b},{ }^{*} ;}$ Jill Lacy, MDc; Andrew Hendifar, $\mathrm{MD}^{\mathrm{d}}$; \\ Khanh Nguyen, $\mathrm{MD}^{\mathrm{e}}$; Jamie Koo, $\mathrm{MD}^{\mathrm{f}}$; Jon $\mathrm{H}$. Chung, $\mathrm{PhD}^{\mathrm{b}}$; Joel Greenbowe, $\mathrm{PhD}^{\mathrm{b}}$; Jeffrey S. Ross, $\mathrm{MD}^{\mathrm{b}, \mathrm{s}}$; \\ Marina N. Nikiforova, MDa; Herbert J. Zeh, $\mathrm{MD}^{\mathrm{h}}$; Inderpal S. Sarkaria, $\mathrm{MD}^{\mathrm{h}}$; Anil Dasyam, MD; \\ and Nathan Bahary, MD, PhD
}

\begin{abstract}
Pancreatic ductal adenocarcinoma (PDAC) is one of the most lethal cancers, with a 5-year survival of $8 \%$. Current therapeutic regimens are largely ineffective and underscore the need for novel treatment strategies. Chromosomal rearrangements involving the anaplastic lymphoma kinase $(A L K)$ gene have been identified in several neoplasms. In addition, ALK protein inhibitors have proven efficacy in patients with $A L K$-rearranged tumors. However, $A L K$ translocations in PDAC have not been described. Through comprehensive genomic profiling of 3,170 PDACs, we identified 5 cases $(0.16 \%)$ that harbored an $A L K$ fusion gene: an exon 6 EML4-exon 20 ALK translocation ( $\mathrm{n}=3$ ), an exon 13 EML4-exon 20 ALK translocation $(n=1)$, and an exon 3 STRN-exon 20 ALK translocation $(n=1)$. Among the most prevalent PDACrelated genes, activating KRAS mutations were absent in all 5 cases, who were $<50$ years of age. Among patients aged $<50$ years in our study cohort, $A L K$ translocations constituted $1.3 \%$ of PDACs. Four of 5 patients were treated with an ALK inhibitor, and 3 of these patients demonstrated stable disease, radiographic response, and/or normalization of serum CA 19-9. Although rare, ALK fusions occur in PDAC, and screening for $A L K$ rearrangements should be considered in young patients with PDAC.
\end{abstract}

J Natl Compr Canc Netw 2017;15(5):555-562

Pancreatic ductal adenocarcinoma (PDAC) is the third leading cause of cancer death in the United States. In 2016, the American Cancer Society estimated that 53,070 individuals would be diagnosed with PDAC and approximately 41,780 would die of this deadly disease. ${ }^{1}$ Although surgical resection offers the only possibility of cure, $>85 \%$ of patients present with inoperable disease at diagnosis. Therefore, chemotherapy is the mainstay of treatment for most patients. Unfortunately, current therapeutic regimens, including gemcitabine plus nabpaclitaxel and FOLFIRINOX (5-fluorouracil, folinic

From a Department of Pathology, University of Pittsburgh Medical Center, Pittsburgh, Pennsylvania; ${ }^{\mathrm{b} F o u n d a t i o n}$ Medicine, Inc, Cambridge, Massachusetts; 'Department of Medicine, Section of Medical Oncology, Yale School of Medicine, New Haven, Connecticut; dDivision of Hematology and Oncology, Cedars-Sinai Medical Center, Los Angeles, California; 'Department of Medicine, University of Pittsburgh Medical Center, Pittsburgh, Pennsylvania; ${ }^{\mathrm{f}}$ Department of Pathology, Cedars-Sinai Medical Center, Los Angeles, California; ${ }^{9}$ Department of Pathology, Albany

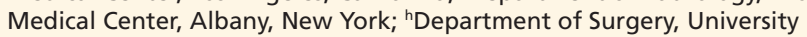
of Pittsburgh Medical Center, Pittsburgh, Pennsylvania; and iDepartment of Radiology, University of Pittsburgh Medical Center, Pittsburgh, Pennsylvania.

*These authors contributed equally. acid, oxaliplatin, and irinotecan), have limited efficacy, with an incremental survival benefit of only a few months in unselected patients. However, significant responses have been observed in small subgroups. ${ }^{2}$

Whole-exome and whole-genome sequencing studies of large PDAC cohorts have revealed a diverse number of genetic alterations in otherwise histologically similar tumors. ${ }^{3,4}$ The intertumoral heterogeneity of molecular abnormalities may partly explain the poor response rates to current therapeutic regimens among unselected patients with PDAC. ${ }^{5}$ Thus, there has been

Submitted January 18, 2017; accepted for publication February 15, 2017.

Drs. Ali, Chung, Greenbowe, and Ross have employment and stock ownership in Foundation Medicine, Inc. The remaining authors have disclosed that they have no financial interests, arrangements, affiliations, or commercial interests with the manufacturers of any products discussed in this article or their competitors.

This study was supported in part by a grant from the National Pancreas Foundation, Western Pennsylvania Chapter, and the University of Pittsburgh (A.D.S.).

Correspondence: Nathan Bahary, MD, PhD, UPMC Cancer Pavilion, 5150 Centre Avenue, 5th Floor, Pittsburgh, PA 15232.

E-mail: baharyn@upmc.edu 
Singhi et al

a recent emphasis on a more personalized approach to the treatment of PDAC based on its underlying genetic alterations. For instance, PDACs harboring mutations in DNA repair genes, such as BRCA2 or PALB2, are often sensitive to poly(ADP-ribose) polymerase inhibitors and cisplatin. ${ }^{6}$ Moreover, mutations in the mismatch repair genes confer susceptibility to immune checkpoint inhibitors. ${ }^{7}$ However, these "actionable" genetic alterations are relatively uncommon and, consequently, there is an urgent need to identify additional molecular targets.

In recent years, chromosomal rearrangements involving the anaplastic lymphoma kinase (ALK) gene have been the subject of intense clinical investigation. ${ }^{8}$ The ALK protein is a receptor tyrosine kinase and physiologically expressed within the central nervous system. Translocation of ALK with various partner genes results in an ALK fusion protein and constitutive ALK activation. Several ALK fusion genes have been reported and are considered drivers of tumorigenesis for a wide range of neoplasms. In addition, the ALK protein inhibitors crizotinib, ceritinib, and alectinib have proven efficacy in patients with ALK-rearranged tumors. ${ }^{9,10}$ Therefore, the ALK fusion protein represents an attractive target for directed therapy, but, to date, has not been reported in PDACs. We examined the prevalence of ALK rearrangements within a large cohort of locally advanced and metastatic PDACs and identified 5 patients with an ALK-rearranged PDAC. We further analyzed their associated clinicopathologic features and molecular profile. Follow-up information was available for 4 patients, including treatment response data with ALK protein inhibitors.

\section{Methods}

\section{Comprehensive Genomic Profiling}

Ten 4-mcm unstained sections from 3,170 PDACs were submitted to a CLIA-certified and College of American Pathologists-accredited laboratory (Foundation Medicine, Inc., Cambridge, MA) for targeted next-generation sequencing and gene fusion detection (see supplemental eTable 1, available with this article at JNCCN.org). ${ }^{11}$ DNA was extracted and quantitated by a PicoGreen fluorescence assay. Thereafter, approximately 50 to $100 \mathrm{ng}$ of DNA was used for library construction. Hybrid selection and sequencing were performed of all coding exons of 295 cancer-associated genes and select introns of 19 genes to include 47 introns frequently rearranged in solid tumors (including ALK). The average depth of coverage was $>500 x$, with $>99 \%$ of exons with $>100 x$ coverage using the Illumina HiSeq 2000 (Illumina, Inc., San Diego, CA). Sequencing data were processed using a customized set of analysis tools, designed to detect all classes of genomic alterations that include base substitutions, short insertions and deletions, copy number alterations, and genomic rearrangements.

\section{ALK Fluorescence In Situ Hybridization}

Fluorescence in situ hybridization (FISH) was assessed using a break-apart probe to the ALK gene (Vysis LSI ALK Dual Color, Break Apart Rearrangement Probe; Abbott Molecular, Des Plains, IL). Staining of tissue sections was performed using 4-mcm unstained paraffin sections. At least 60 tumor nuclei were scored with positivity, defined as $>15 \%$ of tumor cells demonstrating split signals (isolated red signals and/or split red and green signals). Overlapping cells were excluded from analysis.

\section{ALK Immunohistochemistry}

Immunohistochemical labeling was performed on 4-mcm unstained sections. Slides were deparaffinized with serial xylene treatments and subjected to antigen retrieval using heated citrate solution $(\mathrm{pH} 9.0)$ at $100^{\circ} \mathrm{C}$ for 10 minutes. Immunolabeling for ALK (D5F3 rabbit monoclonal, Ventana Medical Systems, Inc., Tucson, AZ) was performed on the automated Benchmark XT system (Ventana Medical Systems, Inc., Tucson, AZ) using the biotin-free Ventana OptiView DAB IHC Detection Kit (Ventana Medical Systems, Inc., Tucson, AZ). Positive cases were defined by the presence of strong granular cytoplasmic staining in at least $10 \%$ of tumor cells.

\section{Results}

Targeted next-generation sequencing of 295 genes and gene fusion detection of 19 genomic rearrangements (supplemental eTable 1) to include ALK were performed on 3,170 PDACs in the course of clinical care. ${ }^{11}$ The patient cohort consisted of 1,724 men and 1,446 women ranging in age from 19 to 88 years (median, 63 years). In most cases, patients were described as having locally advanced and/or metastatic disease. In total, 5 of 3,170 PDACs $(0.16 \%)$ 
harbored an ALK rearrangement. Among the 5 tumors, an exon 13 EML4-exon 20 ALK translocation was detected in 1 case (Case 1), an exon 6 EML4exon 20 ALK translocation in 3 cases (Cases 2, 4, and 5), and an exon 3 STRN-exon 20 ALK translocation in 1 case (Case 3) (Figure 1A-C). The corresponding clinicopathologic features for each patient are summarized in Table 1 . The patients consisted of 4 men and 1 woman ranging in age from 32 to 46 years (mean, 38 years). Among patients $<50$ years of age within the cohort $(n=385)$, ALK translocations were identified in $1.3 \%$. Morphologically, 4 cases were tubular-type PDACs and 1 was a colloid carcinoma (Figure 1D-F). However, among the 4 tubulartype PDACs, 1 showed prominent extracellular mucin. Clinical presentation, treatment, and follow-up information was available for 4 of 5 patients.

\section{Case 1}

A 35-year-old man presented with fatigue, diarrhea, and jaundice. No personal or family history of cancer, pancreatitis, or liver disease was reported. The patient denied a history of tobacco, alcohol, or recreational drug use. Abdominal contrast-enhanced CT showed a $2.1-\mathrm{cm}$ pancreatic head mass, which was confirmed as PDAC through subsequent endoscopic ultrasound (EUS)-guided fine-needle aspiration (FNA). His serum CA 19-9 level was elevated at $54.2 \mathrm{U} / \mathrm{mL}$ (normal $<33 \mathrm{U} / \mathrm{mL}$ ). Sonographic evidence suggested invasion into the portal vein, and the patient was deemed borderline resectable. The patient completed 6 cycles of neoadjuvant FOLFIRINOX with a radiologic decrease in tumor size to $1.8 \mathrm{~cm}$ and CA $19-9$ normalization. Due to chemotoxicity, the patient was switched to gemcitabine and nab-paclitaxel for 2 cycles. This was followed by stereotactic body radiation therapy (RT). After 7 months from initial diagnosis, a robotic-assisted classic pancreaticoduodenectomy (Whipple resection) was performed. The surgical specimen revealed a 2.0-cm moderately differentiated PDAC centered within the pancreatic head and extension into the peripancreatic soft tissue. In addition, 2 of 56 regional lymph nodes were involved via metastatic PDAC. Overall, the PDAC was pathologically staged as ypT3N1. Because of delayed surgical recovery, the patient did not receive adjuvant therapy.

Three months after surgery, the patient's CA 19-9 level was elevated at $56.3 \mathrm{U} / \mathrm{mL}$; however, no evidence of recurrence was identified on CT
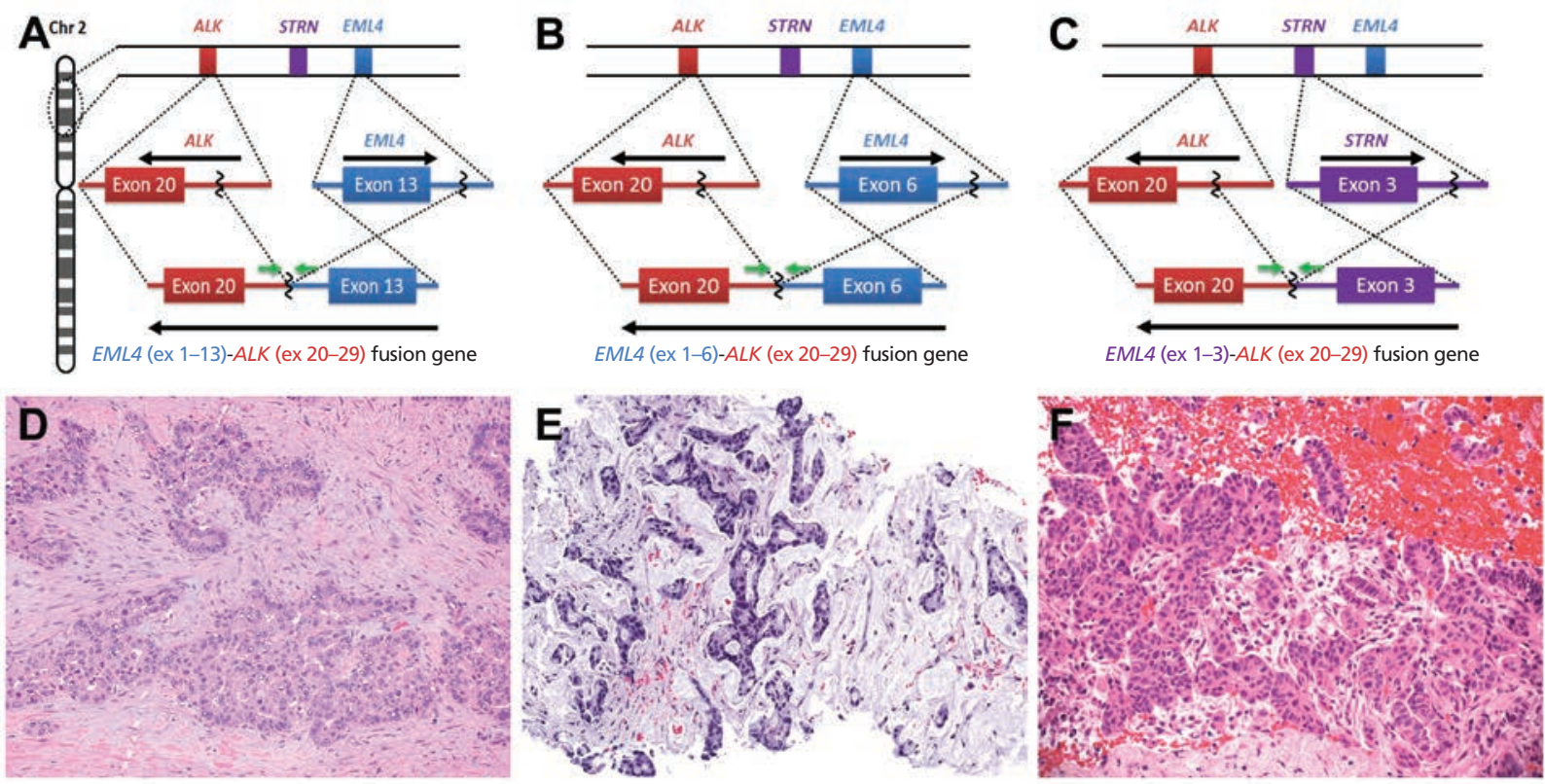

Figure 1. Comprehensive genomic profiling identified $3 A L K$ fusion genes within pancreatic ductal adenocarcinomas (PDACs). The $A L K$ fusion genes consisted of (A) an exon 13 EML4-exon 20 ALK translocation (Case 1), (B) an exon 6 EML4-exon 20 ALK translocation (Cases 2, 4, and 5), and (C) an exon 3 STRN-exon 20 ALK translocation (Case 3). Green arrows depict uniquely mapped paired-end reads (of $\geq 15$ primer pairs) in the intronic regions of EML4 (introns 13 and 6) or STRN (intron 3) and ALK (intron 19). Black arrows indicate the direction of transcription of each gene. Hematoxylin and eosin-stained slides of $A L K$-positive tumors were heterogeneous and consisted of tubular-type (D, Case 1; F, Case 3) and colloid-type (E, Case 5) adenocarcinomas (original magnification $\times 200$ ). 
Singhi et al

\begin{tabular}{|c|c|c|c|c|c|c|c|}
\hline Case & Age, y & Sex & $\begin{array}{l}\text { Histologic } \\
\text { Subtype }\end{array}$ & $\begin{array}{c}\text { Distant } \\
\text { Metastases }\end{array}$ & $\begin{array}{c}A L K \\
\text { Rearrangement }\end{array}$ & $\begin{array}{l}\text { Other Molecular } \\
\text { Alterations } \\
\text { (MAF/CN) }\end{array}$ & $\begin{array}{c}\text { Median Exon } \\
\text { Coverage }\end{array}$ \\
\hline 1 & 35 & $M$ & $\begin{array}{c}\text { Tubular-type } \\
\text { adenocarcinoma }\end{array}$ & Yes & $\begin{array}{c}\text { Exon } 13 E M L 4 \text {-exon } \\
20 A L K\end{array}$ & $\begin{array}{l}\text { BAP1 c.1714delC }(7 \%)^{\mathrm{a}} \\
\text { NFE2L2 c.40C }>\text { T }(5 \%) \\
\text { MCL1 amplification (7) }\end{array}$ & 690 \\
\hline 2 & 32 & $\mathrm{~F}$ & $\begin{array}{l}\text { Tubular-type } \\
\text { adenocarcinoma with } \\
\text { mucinous features }\end{array}$ & Yes & $\begin{array}{c}\text { Exon } 6 \text { EML4-exon } \\
20 A L K\end{array}$ & $\begin{array}{c}\text { CDKN2A/B homozygous } \\
\text { deletion } \\
\text { MYC amplification (82) } \\
\text { TP53 c.325_326insTTCG (59\%) }\end{array}$ & 667 \\
\hline 3 & 34 & M & $\begin{array}{c}\text { Tubular-type } \\
\text { adenocarcinoma }\end{array}$ & No & $\begin{array}{c}\text { Exon } 3 \text { STRN-exon } \\
20 A L K\end{array}$ & None & 896 \\
\hline 4 & 46 & M & $\begin{array}{c}\text { Tubular-type } \\
\text { adenocarcinoma }\end{array}$ & Yes & $\begin{array}{c}\text { Exon } 6 \text { EML4-exon } \\
20 A L K\end{array}$ & $\begin{array}{c}\text { CDKN2A homozygous } \\
\text { deletion } \\
\text { TP53 c.994-1G>C (51\%) } \\
\text { SMAD4 c.247C >T (51\%) } \\
\text { FGFR1 c.422C>G (65\%) }\end{array}$ & 734 \\
\hline 5 & 43 & M & Colloid carcinoma & Unknown & $\begin{array}{c}\text { Exon } 6 \text { EML4-exon } \\
20 A L K\end{array}$ & STK11 c.226delG (7\%) & 618 \\
\hline
\end{tabular}

Abbreviations: $\mathrm{CN}$, copy number; MAF, mutation allele frequency.

${ }^{a} B A P 1$ mutation was present within the patient's primary tumor; however, a PBRM1 mutation was identified within the patient's subsequent lung metastasis.

imaging. At 11 months, the patient's serum CA 19-9 level had increased to $584.9 \mathrm{U} / \mathrm{mL}$. CT imaging revealed enlarged retroperitoneal (left periaortic and retrocaval) and posterior mediastinal lymph nodes consistent with metastatic disease. Gemcitabine and nab-paclitaxel were administered for 3 cycles, but treatment was complicated by chemotoxicity and had to be stopped.

Comprehensive genomic profiling (CGP) of the patient's primary PDAC revealed an exon 13 EML4-exon 20 ALK translocation, which was confirmed with FISH using a break-apart probe for ALK (Figure 2A) and ALK immunohistochemistry (Figure 2B). Additionally, a BAP1 frameshift mutation (c.1714delC, p.L573fs*3), NFE2L2 missense mutation (c.40C>T), and MCL1 gene amplification were identified. Considering the unique genetic alterations, additional immunohistochemical stains were performed on the patient's PDAC. The tumor immunolabeled for CK7 but was negative for CK20, CDX2, TTF-1, napsin A, PAX8, GCDFP-15, mammaglobin, estrogen receptor (ER), and progesterone receptor (PR). Based on the clinical, radiographic, pathologic, and immunolabeling findings, the tumor was consistent with a PDAC.

One month after discontinuing gemcitabine and nab-paclitaxel, the patient was started on crizotinib, but experienced neutropenia and was switched to ceritinib. Within 2 months, his CA 19-9 level normalized (23.5 U/mL; Figure 3A). Moreover, there was a decrease in the size of retroperitoneal and pos- terior mediastinal lymph nodes (Figure 3B-E). After 7 months on ceritinib, the patient's CA 19-9 level increased to $79.8 \mathrm{U} / \mathrm{mL}$ and correlated with the development of a $0.8-\mathrm{cm}$ right upper lobe lung nodule. However, the patient's intra-abdominal disease remained stable. A wedge resection of the lung nodule was performed and was consistent with metastatic PDAC. Repeat genetic profiling continued to demonstrate an exon 13 EML4-exon 20 ALK translocation, NFE2L2 missense mutation, and MCL1 gene amplification, but lacked a BAP1 frameshift mutation. Instead, a frameshift mutation in PBRM1 (c.3558_3559insA, p.E1189fs*6) was identified. ALK secondary mutations and gene amplification were absent. Further, no alterations in KRAS were detected. The patient was switched to alectinib and is alive and well at the time of writing; the duration of survival from diagnosis is currently 52 months.

\section{Case 2}

A 32-year-old woman presented with increasing fatigue, shortness of breath, recurrent nausea and vomiting, lightheadedness, and distal upper extremity paresthesia. The patient denied a history of other cancers. CT imaging identified multiple masses within the pancreas, liver, ovaries, and peritoneum. The largest mass was centered within the head of the pancreas. An exploratory laparotomy confirmed the presence of a large pancreatic head mass and multiple smaller lesions within the liver, ovaries, and peritoneum. Resection of multiple liver lesions and bilateral 

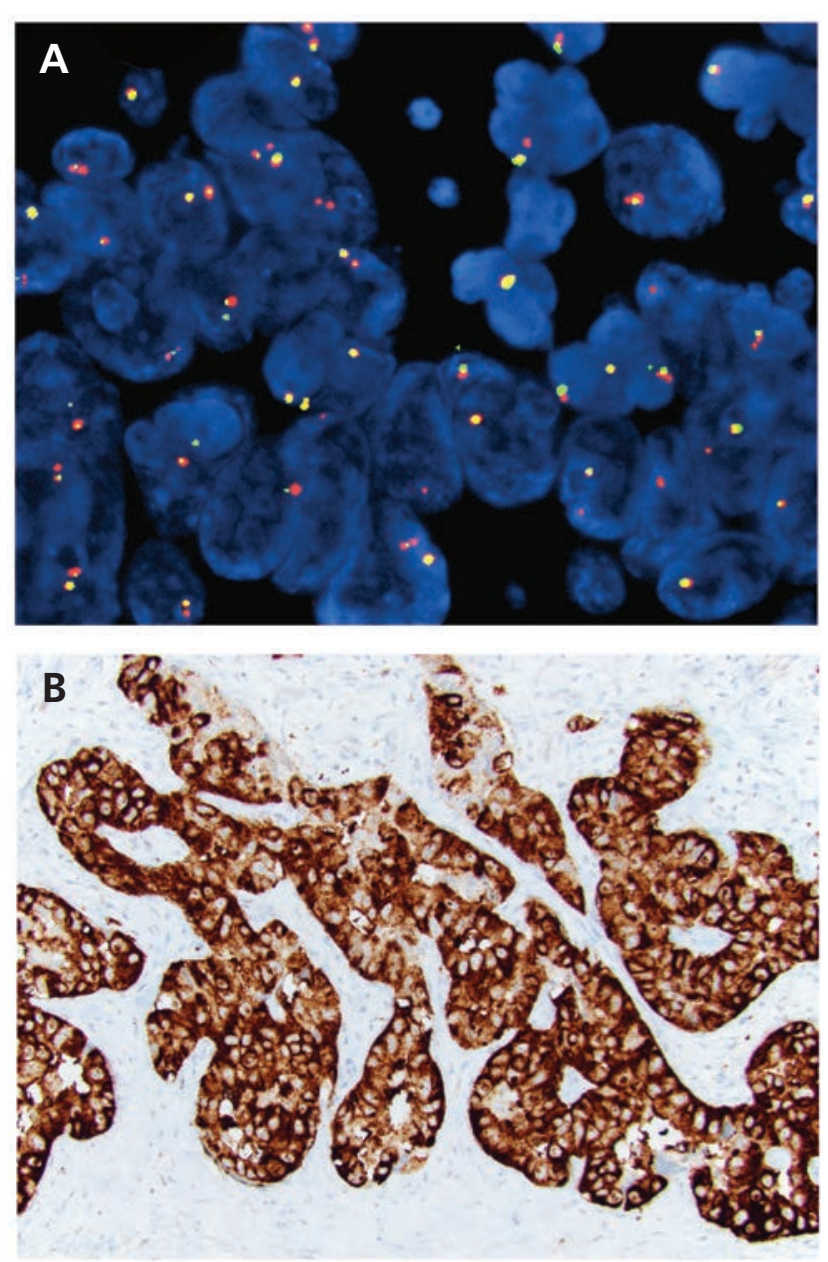

Figure 2. Both Case 1 (exon 13 EML4-exon 20 ALK fusion gene) and Case 3 (exon 3 STRN-exon 20 ALK fusion gene) were confirmed to harbor an $A L K$ rearrangement by (A) $A L K$ break-apart fluorescence in situ hybridization probe, which demonstrated isolated red signals in $>15 \%$ of neoplastic nuclei (original magnification $x 1,000$ ), and (B) ALK immunohistochemistry, which showed strong, diffuse, and granular cytoplasmic staining (original magnification $\times 400$ ).

salpingo-oophorectomy was performed for pathologic evaluation and ancillary studies. The specimens revealed a moderately differentiated adenocarcinoma with prominent mucinous features. Immunohistochemical stains were strongly positive for CK7 and CA 19.9, and negative for CK20, PAX8, CDX2, ER, and PR. Additionally, Smad4, MLH1, PMS2, MSH2, and MSH6 immunolabeling showed preserved nuclear expression. Overall, the histologic features and immunoprofile were consistent with a pancreatic primary.

Adjuvant therapy consisted of FOLFIRINOX for 2 cycles with subsequent chemotoxicity. The patient was switched to gemcitabine and nab-paclitaxel, but again experienced chemotoxicity, and progression of her liver metastases was seen on MRI. CGP of one of her metastases revealed an exon 6 EML4-exon 20 ALK translocation, CDKN2A/B deletion, MYC gene amplification, and TP53 frameshift mutation (c.325_326insTTCG, p.L111fs*39). Based on these findings, the patient was started on crizotinib alone, and 2 months later was found to have stable metastatic disease on MRI. The primary site and metastases remained stable on follow-up MRI for the next 15 months. However, the patient's clinical course was complicated by repeated episodes of bacteremia and, ultimately, she died of septic shock. The duration of survival from diagnosis was 20 months.

\section{Case 3}

A 34-year-old man presented with progressive fatigue, pale skin, and anemia. The patient denied a personal or family history of cancer, pancreatitis, or liver disease. In addition, the patient denied a history of tobacco, alcohol, or recreational drug use. An abdominal CT identified a $7.5-\mathrm{cm}$ pancreatic mass involving the superior mesenteric artery and vein. No evidence of distant metastatic disease was seen. A follow-up EUS-FNA confirmed the presence of a pancreatic head mass with secondary biliary obstruction, and cytopathology was consistent with a PDAC. The patient received 4 cycles of FOLFIRINOX with stable-to-slight disease progression by CT. Further, the patient's serum CA 19-9 level was elevated at $410 \mathrm{U} / \mathrm{mL}$.

CGP of the pancreatic head biopsy revealed the presence of an exon 3 STRN-exon 20 ALK translocation; no other genetic alterations were identified. The presence of an ALK translocation was confirmed by ALK break-apart FISH and ALK immunohistochemistry. The patient began treatment with gemcitabine and crizotinib, and also received externalbeam RT. After 2 months of chemoradiotherapy, his serum CA 19-9 level decreased to $84 \mathrm{U} / \mathrm{mL}$. Further, repeat abdominal CT showed a decrease in tumor size to $6.9 \mathrm{~cm}$. The patient is alive and continuing only crizotinib therapy at the time of writing. The duration of survival from diagnosis is currently 10 months.

\section{Case 4}

Limited clinical information was available for Case 4, a 46-year-old man who presented with a locally advanced pancreatic head mass consistent with PDAC. An exon 6 EML4-exon 20 ALK translocation 
Singhi et al

was identified through CGP of biopsy material from the pancreatic head. Additional genetic alterations included a homozygous CDKN2A deletion, TP53 splice site mutation (c.994-1G>C), SMAD4 nonsense mutation (c.247C>T, p.Q83*), and FGFR1 missense mutation (c.422C>G, p.T141R). The patient was treated with crizotinib alone, but experienced progression after 2 months, with development of leptomeningeal disease and secondary spinal cord compression. The patient received external-beam RT to his thoracic spine and was started on alectinib. The duration of survival from diagnosis is 5 months at the time of writing.

\section{Discussion}

To the best of our knowledge, this is the first report of targetable ALK translocations in PDAC. Among 3,170 PDACs, we identified 5 cases harboring either an EML4-ALK or STRN-ALK fusion gene. The patients consisted of 4 men and 1 women ranging in age from 32 to 46 years. Morphologically, the ALK-rearranged PDACs were classified as either tubular or colloid, but among the tubular cases, 1 exhibited prominent extracellular mucin. Besides the presence of an $A L K$ rearrangement, other genetic abnormalities included somatic alterations in BAP1, CDKN2A/B, FGFR1, MCL, MYC, NFE2L2, SMAD4, STK11, and TP53. Of note, the most frequently mutated gene in PDAC is KRAS. However, KRAS mutations were lacking in all ALK-rearranged PDACs.

ALK fusion genes, including EML4-ALK and STRN-ALK, have been identified in multiple cancer types, including lung adenocarcinoma, colorectal adenocarcinoma, breast cancer, renal cell carcinoma, renal medullary carcinoma, and papillary thyroid carcinoma. ${ }^{12-15}$ Based on previous reports, the resulting chimeric proteins possess potent oncogenic activity in both in vitro and in vivo models. ${ }^{8,16,17}$ In lung adenocarcinoma, ALK fusions are detected in approximately $1 \%$ of tumors and are often characterized by distinct epidemiologic, demographic, and pathologic features. ALK-positive lung adenocarcinomas typically present at a young age, and are often characterized by prominent mucinous cells, such as signet-ring cells and goblet cells, and abundant extracellular mucin. ${ }^{18}$ Further, rearrangements involving ALK in lung adenocarcinomas are mutually exclusive to mutations in KRAS and EGFR. ${ }^{19}$
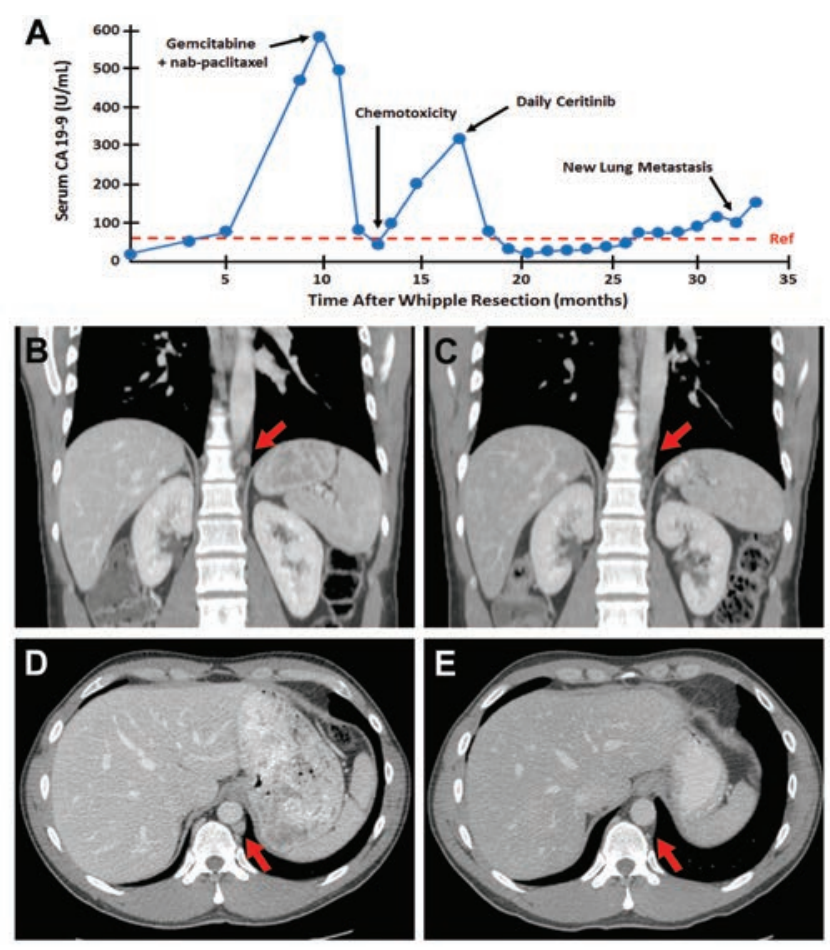

Figure 3. The clinical and treatment course of Case 1 after Whipple (classic pancreaticoduodenectomy) resection. (A) An increase in serum CA 19-9 levels was associated with disease recurrence and the disease initially responded to gemcitabine and nab-paclitaxel. The patient soon developed chemotoxicity, which necessitated cessation of therapy. On identification of an exon 13 EML4-exon 20 ALK translocation, daily ceritinib alone was started and a decrease in serum CA 19-9 levels was observed shortly thereafter. However, the patient's serum CA 19-9 levels slowly increased over the next several months with the development of a new lung metastasis. $(B, D)$ Before ceritinib treatment, CT scan for Case 1 showed an enlarged posterior mediastinal lymph node (red arrow). (C, E) Within 2 months of treatment, a repeat CT scan showed resolution of the patient's posterior mediastinal lymph node (red arrow).

Analogous to lung adenocarcinoma, ALK rearrangements in PDAC were detected in a small percentage of cases, with a prevalence of $0.16 \%$. Indeed, considering the low prevalence of ALK translocations, many reports have concluded that rearrangements in $A L K$ are absent in PDAC. ${ }^{20}$ Moreover, a striking feature of ALK-positive PDACs is a young age at onset, with a mean age of 38 years. In comparison, the mean age of patients with PDAC is 65 years. ${ }^{21}$ In contrast to ALK-rearranged lung adenocarcinomas, signet-ring cells and goblet cells were absent in ALK-positive PDACs; however, abundant extracellular mucin was identified in 2 cases. Lastly, KRAS mutations are nearly ubiquitous in PDAC, but none of the ALK-translocated PDACs harbored a mutation in KRAS, which may reflect the mutual exclusivity of these alterations. The similarities between ALK- 
positive PDACs and lung adenocarcinoma as well as other ALK-translocated carcinomas are intriguing and suggest not only a common pathogenesis, but possibly an equivalent response to treatment.

Several studies have demonstrated that ALKrearranged tumors are highly responsive to small molecule tyrosine kinase inhibitors, such as crizotinib and ceritinib. However, despite the initial sensitivity, most patients will eventually experience relapse within 12 months. ${ }^{22}$ Mechanisms of acquired resistance include secondary mutations in the ALK tyrosine kinase domain, ALK fusion gene amplification, and activation of alterative tyrosine kinases or downstream genes, such as KRAS mutations. ${ }^{23}$ Consistent with these findings, on treatment with ALK inhibitors, 3 of 4 patients described herein demonstrated either stable disease or radiographic response with normalization of serum CA 19-9 levels. For Case 1, within 2 months of starting ceritinib, both a radiographic and serum CA 19-9 response were identified, but after 7 months resistance was observed. However, the pattern of ceritinib resistance was heterogeneous in distribution, with development of a new metastasis within the lung and stable intraabdominal disease. CGP of the patient's primary tumor and subsequent lung metastasis revealed discordant results. Before ceritinib treatment, in addition to an EML4-ALK translocation, a BAP1 mutation was identified within the patient's primary PDAC. In contrast, the patient's pulmonary metastasis was wild-type for BAP1 but harbored a PBRM1 mutation. No ALK secondary mutations, EML4-ALK gene amplification, or other genetic alterations associated with receptor tyrosine kinase pathway activation were detected within the pulmonary metastasis.

PBRM1 is hypothesized to be a tumor suppressor gene and encodes for the protein BAF180, a component of the SWI/SNF chromatin remodeling complex that regulates gene expression through nucleosome repositioning. ${ }^{24}$ Loss of PBRM1 is associated with increased cell proliferation and motility. PBRM1 is believed to have regulatory effects on TP53, CDKN1A/p21, and the KRAS pathway. In fact, Varela et $\mathrm{al}^{25}$ found PBRM1 inactivation cooperates with mutant KRAS in driving tumor development in a mouse model of PDAC. Considering the possible mutual exclusivity between KRAS mutations and ALK rearrangements in PDAC, it is plausible that a PBRM1 inactivation can potentiate the downstream effects of ALK tyrosine kinase activation. Moreover, mutations in PBRM1 and BAP1 with loss of heterozygosity of the wild-type allele tend to be subclonal and mutually exclusive, which could explain the lack of a BAP1 inactivation within the patient's pulmonary metastasis. ${ }^{26}$

It is important to note that there are a number of limitations in this study. Although all patients within our cohort were clinically and pathologically considered to have PDAC, confirmatory surgical resection material was only available for a single patient with an ALK-translocated PDAC. However, $85 \%$ patients with PDAC present with locally advanced and/or metastatic disease. Regardless, the lack of confirmatory resection specimens does not minimize the significance of our findings. Three of 4 patients with ALK translocations within our cohort demonstrated stable disease, radiographic response, and/or normalization of serum CA 19-9 to ALK inhibitors. In addition, the low prevalence of ALK translocations in PDAC may be viewed as insignificant to justify the expense of CGP. The young age at presentation for patients with ALK-positive PDAC may be used to screen patients for these targetable translocations. In fact, among patients $<50$ years of age within our study cohort, $1.3 \%$ of patients with PDAC harbored an $A L K$ translocation. Further, similar to lung adenocarcinoma, low-cost alternatives, such as ALK breakapart FISH and ALK immunohistochemistry, may be used to screen for ALK rearrangements in PDAC.

\section{Conclusions}

Rearrangements in ALK are present in a small subset of PDACs. Similar to other ALK translocated cancers, PDACs with ALK translocations are characterized by young patient age at presentation, an absence of KRAS mutations, and a clinical response to ALK inhibitors. Considering the low prevalence of $A L K$ rearrangements in PDAC, screening for these "actionable" targets may be facilitated by focusing on patients presenting with PDAC at an age of $<50$ years.

\section{Acknowledgments}

The authors would like to thank Mrs. Robyn L. Roche for outstanding administrative assistance. 
Singhi et al

\section{References}

1. American Cancer Society. Key Statistics for Pancreatic Cancer. Available at: http://www.cancer.org/cancer/pancreaticcancer/detailedguide/pancreaticcancer-key-statistics. Accessed April 4, 2017.

2. Biankin $A V$, Hudson TJ. Somatic variation and cancer: therapies lost in the mix. Hum Genet 2011;130:79-91.

3. Waddell N, Pajic M, Patch AM, et al. Whole genomes redefine the mutational landscape of pancreatic cancer. Nature 2015;518:495-501.

4. Jones $\mathrm{S}$, Zhang $\mathrm{X}$, Parsons DW, et al. Core signaling pathways in human pancreatic cancers revealed by global genomic analyses. Science 2008;321:1801-1806.

5. Samuel N, Hudson TJ. The molecular and cellular heterogeneity of pancreatic ductal adenocarcinoma. Nat Rev Gastroenterol Hepatol 2012;9:77-87.

6. Lowery MA, Kelsen DP, Stadler ZK, et al. An emerging entity: pancreatic adenocarcinoma associated with a known BRCA mutation: clinical descriptors, treatment implications, and future directions. Oncologist 2011;16:1397-1402.

7. Le DT, Uram JN, Wang H, et al. PD-1 blockade in tumors with mismatchrepair deficiency. N Engl J Med 2015;372:2509-2520.

8. Mano H. ALKoma: a cancer subtype with a shared target. Cancer Discov 2012;2:495-502.

9. Shaw AT, Yeap BY, Solomon BJ, et al. Effect of crizotinib on overall survival in patients with advanced non-small-cell lung cancer harbouring ALK gene rearrangement: a retrospective analysis. Lancet Oncol 2011;12:1004-1012.

10. Friboulet L, Li N, Katayama R, et al. The ALK inhibitor ceritinib overcomes crizotinib resistance in non-small cell lung cancer. Cancer Discov 2014;4:662-673.

11. Frampton GM, Fichtenholtz A, Otto GA, et al. Development and validation of a clinical cancer genomic profiling test based on massively parallel DNA sequencing. Nat Biotechnol 2013;31:1023-1031.

12. Lin E, Li L, Guan Y, et al. Exon array profiling detects EML4-ALK fusion in breast, colorectal, and non-small cell lung cancers. Mol Cancer Res 2009; 7:1466-1476.
13. Kelly LM, Barila G, Liu P, et al. Identification of the transforming STRNALK fusion as a potential therapeutic target in the aggressive forms of thyroid cancer. Proc Natl Acad Sci U S A 2014;111:4233-4238.

14. Soda M, Choi YL, Enomoto M, et al. Identification of the transforming EML4-ALK fusion gene in non-small-cell lung cancer. Nature 2007;448:561-566.

15. Marino-Enriquez $A, O u$ WB, Weldon $C B$, et al. ALK rearrangement in sickle cell trait-associated renal medullary carcinoma. Genes Chromosomes Cancer 2011;50:146-153.

16. Soda M, Takada S, Takeuchi K, et al. A mouse model for EML4-ALKpositive lung cancer. Proc Natl Acad Sci U S A 2008;105:19893-19897.

17. McDermott U, Iafrate AJ, Gray NS, et al. Genomic alterations of anaplastic lymphoma kinase may sensitize tumors to anaplastic lymphoma kinase inhibitors. Cancer Res 2008;68:3389-3395.

18. Yoshida A, Tsuta K, Nakamura H, et al. Comprehensive histologic analysis of ALK-rearranged lung carcinomas. Am J Surg Pathol 2011;35:1226-1234.

19. Gainor JF, Varghese AM, Ou SH, et al. ALK rearrangements are mutually exclusive with mutations in EGFR or KRAS: an analysis of 1,683 patients with non-small cell lung cancer. Clin Cancer Res 2013;19:4273-4281.

20. Graham RP, Oliveira AM, Zhang L. Rare ALK expression but no ALK rearrangement in pancreatic ductal adenocarcinoma and neuroendocrine tumors. Pancreas 2013;42:949-951.

21. Singhi AD, Ishida $\mathrm{H}, \mathrm{Ali} \mathrm{SZ}$, et al. A histomorphologic comparison of familial and sporadic pancreatic cancers. Pancreatology 2015;15:387-391.

22. Katayama R, Lovly CM, Shaw AT. Therapeutic targeting of anaplastic lymphoma kinase in lung cancer: a paradigm for precision cancer medicine. Clin Cancer Res 2015;21:2227-2235.

23. Doebele RC, Pilling AB, Aisner DL, et al. Mechanisms of resistance to crizotinib in patients with ALK gene rearranged non-small cell lung cancer. Clin Cancer Res 2012;18:1472-1482.

24. Brugarolas J. Molecular genetics of clear-cell renal cell carcinoma. J Clin Oncol 2014;32:1968-1976.

25. Varela I, Tarpey P, Raine K, et al. Exome sequencing identifies frequent mutation of the SWI/SNF complex gene PBRM1 in renal carcinoma. Nature 2011;469:539-542.

26. Brugarolas J. PBRM1 and BAP1 as novel targets for renal cell carcinoma. Cancer J 2013;19:324-332. 